\title{
Effect of Soft Segments of Waterborne Polyurethane on Organic Vapor Sensitivity of Carbon Black Filled Waterborne Polyurethane Composites
}

\author{
Bin ZhaO, ${ }^{1}$ Ruo Wen Fu, ${ }^{2}$ Ming Qiu Zhang, ${ }^{2, \dagger}$ Hong YANG, ${ }^{1}$ \\ Min Zhi Rong, ${ }^{2}$ and Qiang $\mathrm{ZHENG}^{3}$ \\ ${ }^{1}$ Key Laboratory for Polymeric Composite and Functional Materials of Ministry of Education, \\ Zhongshan University, Guangzhou 510275, P. R. China \\ ${ }^{2}$ Materials Science Institute, Zhongshan University, Guangzhou 510275, P. R. China \\ ${ }^{3}$ Department of Polymer Science and Engineering, Zhejiang University, Hangzhou 310027, P. R. China
}

(Received January 6, 2006; Accepted April 4, 2006; Published July 7, 2006)

\begin{abstract}
In the present work, three types of waterborne polyurethane (WPU) were synthesized, in which poly(ethylene oxalate glycol), poly(propylene oxide) and poly(tetramethylene ether glycol) acted as the soft segments, respectively. The WPUs were then compounded with carbon black (CB) to fabricate gas sensing composites. By studying the composites' resistance variation in different solvent-vapors, it was found that the structures of the WPUs remarkably affected the composites' response behaviors. Both polarity and flexibility of the soft segments of WPU determine to a great extent the solvent-polymer interaction and preferential localization of solvent and fillers, resulting in different magnitudes of resistance increase and rates of response. [doi:10.1295/polymj.PJ2005202]

KEY WORDS Waterborne Polyurethane / Conductive Polymer Composites / Vapor Sensitivity / Structure-Property Relationship /
\end{abstract}

Recently carbon black (CB) filled polymer composites have been exploited for the fabrication of chemical sensors. ${ }^{1,2}$ When being exposed to organic vapors, the composites exhibit a drastic increase in their electrical resistivity because matrix swelling induced by solvents absorption expands the inter-filler gap. ${ }^{3,4}$ Removal of the stimuli leads to desorption of the vapors and a decrease in the composites' resistance back to the original value. On the basis of this feature, therefore, low cost organic solvent leak detectors and "electronic noses" can be manufactured. ${ }^{5-7}$

In the authors' lab, waterborne polyurethane (WPU) was employed to be mixed with CB for preparing gas sensing composites. ${ }^{8}$ As WPU is a segmented polymer consisting of the alternating sequence of long non-polar soft segments and short polar hard segments that constitute a unique microphase separation structure, its composites with $\mathrm{CB}$ exhibit widespectrum responsivity to both low/non-polar and polar solvents.9-12 It was found that the segmented microstructure of WPU greatly influenced the relationships between solvent adsorption behavior and electrical resistance variation of WPU based composites. $^{13}$

In this context, it is worth studying the effect of soft segments on vapor sensitivity of CB/WPU composites, which might lead to optimization of the polymer's microstructure and hence the sensing performance of the composites. Accordingly, three kinds of
WPU were synthesized in the present work, in which only the soft segments are different. These soft segments were selected because of their distinct structures and features. As shown in Table I, poly(tetramethylene ether glycol) (PTMEG) possesses the highest flexibility because there is no side groups on its skeleton, while poly(ethylene oxalate glycol) (PEOG) has the lowest flexibility due to the strong interaction between the ester groups. In regard to polarity, however, the above rank has to be reversed. By comparing electrical response habits of the composites based on these WPUs in benzene (non-polar solvent), chloroform (low polar solvent) and acetone (polar solvent) vapors, it is believed that the influence of WPU microstructure on composites sensitivity would be revealed.

\section{EXPERIMENTAL}

\section{Materials}

Analytically pure ethylenediamine anhydrous (EDA), triethylamine (TEA) and dibutyltin dilaurate were dehydrated via $0.4 \mathrm{~nm}$ molecular sieves for more than one week prior to the experiments. Dimethylolpropionic acid (DMPA) was dried in vacuum at $80^{\circ} \mathrm{C}$ for $24 \mathrm{~h}$. Isophorone diisocyanate (IPDI) was used without further purification. Carbon black (type XC72 , specific surface area $=254 \mathrm{~m}^{2} / \mathrm{g}$, dibutylphthalate absorption $(\mathrm{DPB})$ value $=174 \mathrm{~mL} / 100 \mathrm{~g}$, particle

${ }^{\dagger}$ To whom correspondence should be addressed (Tel: +86-20-84036576, Fax: +86-20-84036576, E-mail: ceszmq@zsu.edu.cn). 
Table I. Soft segments used for synthesizing WPU in this work

\begin{tabular}{ccc}
\hline Soft segment & Structure & $\mu^{*}$ (Debye) \\
\hline Poly(ethylene oxalate glycol) (PEOG) & $\left.\mathrm{H}-\mathrm{O}-\mathrm{C}-\mathrm{C}-\mathrm{O}-\mathrm{CH}_{2}-\mathrm{CH}_{2}\right]_{\mathrm{n}} \mathrm{OH}$ & 0.93 \\
Poly(propylene oxide) (PPG) & $\left.\mathrm{H}-\mathrm{O}-\mathrm{CH}_{2}-\mathrm{CH}\right]_{\mathrm{n}} \mathrm{OH}$ & 0.42 \\
Poly(tetramethylene ether glycol) (PTMEG) & $\left.\mathrm{H}+\mathrm{O}-\left(-\mathrm{CH}_{2}\right)_{4}\right]_{\mathrm{n}} \mathrm{OH}$ & 0.42 \\
\hline
\end{tabular}

${ }^{*} \mu$ : dipole moments of soft segment estimated by using the group contribution method. ${ }^{14}$

size $=50-70 \mathrm{~nm}$ ), purchased from Cabot Co., was dried in vacuum at $110^{\circ} \mathrm{C}$ for $48 \mathrm{~h}$ before use.

\section{Synthesis of WPUs}

Three kinds of oligomer, PEOG, PPG and PTMEG, were used as soft segments (Table I), respectively. The procedures for synthesizing WPU are described as follows. $10 \mathrm{mmol}$ of the oligomer (molecular weight $=2,000$ ) was heated up to $120^{\circ} \mathrm{C}$ in vacuum for $1 \mathrm{~h}$, and then the system was filled up with nitrogen. When the temperature is cooled to $80^{\circ} \mathrm{C}, 6$ droplets of the catalyst (dibutyltin dilaurate) and $30 \mathrm{mmol}$ IPDI were added into the mixture. After $3 \mathrm{~h}$ of reaction, $10 \mathrm{mmol}$ DMPA, dissolved by $10 \mathrm{~mL}$ butanone, was drip-fed into the mixture. The reaction proceeded for $3 \mathrm{~h}$. When the system was cooled to room temperature, the pre-polymer reacted with $10 \mathrm{mmol}$ TEA for $1 \mathrm{~h}$. Then, $100 \mathrm{~mL}$ distilled water and $10 \mathrm{mmol}$ EDA were added to the pre-polymer with high-speed stirring. Twelve hours later, an emulsion of WPU was obtained. For the convenience of discussion, the WPUs synthesized from PEOG, PPG and PTMEG, were denoted by IPDI-DMPA-EDA-PEOG-2000 (WPU-1), IPDI-DMPA-EDA-PPG-2000 (WPU-2) and IPDI-DMPA-EDA-PTMEG-2000 (WPU-3), respectively.

\section{Preparation and Characterization of $C B / W P U$ Com- posites}

For producing conductive composites, a certain amount of $\mathrm{CB}$ particles was added to the above WPU emulsion, and then the mixture was agitated by ultrasonic for $0.5 \mathrm{~h}$ and stirred for additional $4 \mathrm{~h}$. Eventually, the pasty composites were coated onto an epoxy plate with comb electrodes. Prior to further testing, the CB/WPU composite films were dried at room temperature for $2 \mathrm{~d}$ and in vacuum for $2 \mathrm{~d}$, respectively. As the percolation thresholds of all the three CB/WPU composites are lower than $1.5 \mathrm{wt} \%$, the CB content of the WPU composites used in this work is fixed at $4.0 \mathrm{wt} \%$.

Electrical resistance variation of the composites in response to various solvent vapors was measured as

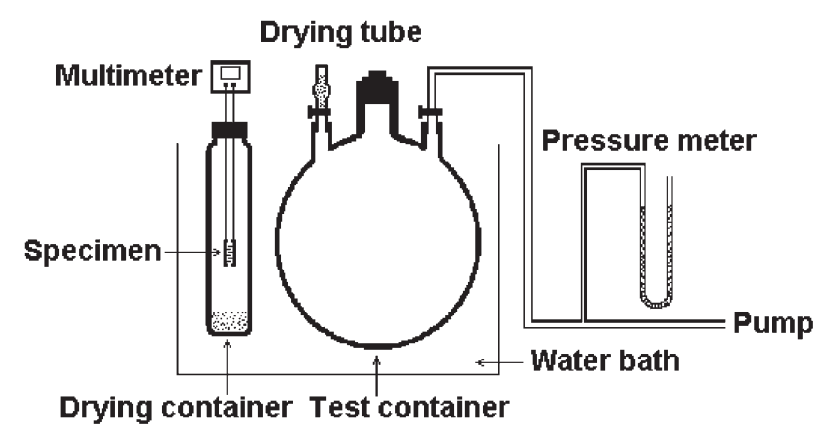

Figure 1. Set-up for measuring electric resistance variation of the composite specimens in response to solvent vapor.

follows. A given volume of solvent was injected into the test container in advance. After ten minutes, the comb electrodes with the composite film $(40-60 \mu \mathrm{m}$ thick), hung in the drying container before test, were rapidly transferred to the test container. It is testified that the change in composites' resistance is neglegible during the transfer. Then, time dependence of resistance of the specimen was recorded by a UT70C digital multimeter (Figure 1). The vapor pressure was calculated by the thermodynamic equation of perfect gas, and the saturated vapor pressure was calculated in terms of Antoine equation. The corresponding responsivity is characterized by $\left(R_{t}-R_{o}\right) / R_{o}$, where $R_{t}$ denotes the transient resistance and $R_{o}$ the initial one in dry air. The maximum responsivity is given by $\left(R_{\max }-R_{o}\right) / R_{o}$, where $R_{\max }$ denotes the maximum resistance.

Solvent vapor adsorption behaviors of the composites were determined by an intelligent gravity analyzer (IGA-03, Hiden Co.). The precision of the system is about $0.1 \mu \mathrm{g}$. The normalized adsorbance is estimated by $M_{t} / M_{e}$, where $M_{t}$ and $M_{e}$ denote the absorbed masses of the specimen at transient and equilibrium states, respectively.

A Netzsch DSC 204 machine was used to record the differential scanning calorimetric (DSC) curves of the WPUs. The experiments were carried out from $-120^{\circ} \mathrm{C}$ to $20^{\circ} \mathrm{C}$ at a heating rate of $20^{\circ} \mathrm{C} /$ min under the protection of $\mathrm{N}_{2}$. 

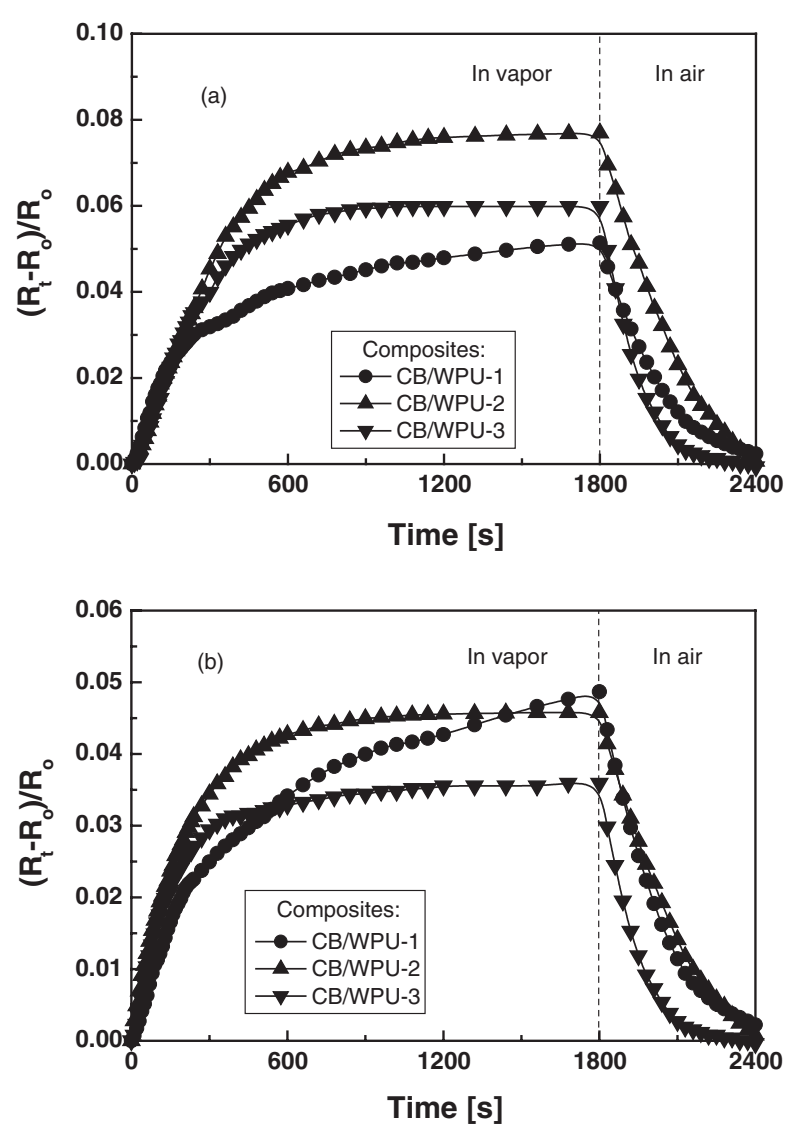

Figure 2. Typical electrical responses of CB/WPU composites against (a) benzene and (b) acetone vapors at a relative pressure of 0.01 (temperature $=35^{\circ} \mathrm{C}$ ). The dash lines define the vapor absorption and desorption zones.

\section{RESULTS AND DISCUSSION}

\section{Solvent-Polymer Interaction}

Typical response behaviors of the CB/WPU composites are shown in Figure 2. The composites are capable of sensing vapors of different polarities. It is noted that different vapors lead to different ways of resistance variation for each composite specimen. In addition, introduction of different soft segments into the molecules of the matrix polymer results in different responsivity even in the case of identical solvent vapor. In fact, magnitude of resistance change of a composite in response to organic vapor is determined not only by the amount of adsorbed solvent, ${ }^{15,16}$ but also by the interaction between the adsorbed solvent molecules and the composite. ${ }^{13}$ When CB loading in the composites is relatively low (4 wt \% in the present $\mathrm{CB} / \mathrm{WPU}$, for example), adsorption of solvent gases of the composites has to be mainly controlled by the matrix polymer. ${ }^{13,16}$ Therefore, the interaction between the adsorbed solvent molecules and the composites can be represented by the interaction between the adsorbed solvent molecules and the chain segments of matrix polymer, regardless of the contribution of CB.

Considering that soft segments are in the majority of WPU, the interactions between the soft segments and solvent molecules should reflect the interactions between the WPUs and solvent molecules when the structure and ratio of hard segments are fixed. That is, the different response manners of the CB/WPU composites illustrated in Figure 2 might be understood by analyzing the solvent-soft segments interactions. For this purpose, Flory-Huggins theory ${ }^{17,18}$ is applied to correlate the sorption isotherms:

$$
\ln \alpha=\ln \phi_{1}+\left(1-\frac{1}{x}\right) \phi_{2}+\chi_{12} \phi_{2}^{2}
$$

where $\alpha$ denotes the activity of vapor, $\phi_{1}$ and $\phi_{2}$ denote the volume fractions of the adsorbed solvent vapor and the matrix polymer (supposing $\phi_{1}+\phi_{2}=1$ ), respectively. $\chi_{12}$ is the Flory-Huggins interaction parameter of the adsorbed solvent vapor and the matrix polymer, and $x$ is the ratio of the molar volumes of the matrix polymer and the adsorbed solvent vapor. In the case of low vapor pressure, the adsorption capacity of the matrix polymer has to be so low that $\phi_{2}$ can be regarded as 1 , and the activity of vapor, $\alpha$, is approximately equal to the relative vapor pressure, $p / p^{o}$. Accordingly, eq 1 is converted to:

$$
\frac{V_{1(\max )}}{V_{o}}=e^{-\left(1+\chi_{12}\right)} \frac{p}{p^{o}}
$$

where $V_{1(\max )}$ and $V_{o}$ denote the maximum volume of the vapor adsorbed by the composite under certain pressure and the initial volume of the composite. From eq 2, it is seen that the equilibrium adsorption capacity of the composites, $V_{1(\max )} / V_{o}$, is proportional to the relative vapor pressure at a certain temperature, and the slope of the linear regression equals to $e^{-\left(1+\chi_{12}\right)}$. To verify this estimation, adsorption experiments in acetone, chloroform and benzene vapors were carried out, and the dependences of equilibrium adsorption capacity on relative pressure are plotted in Figures 3a, 4a and 5a, respectively. Clearly, the linear relationship shown in eq 2 well fits the experimental data, which proves the above deduction. On the basis of this analysis, the Flory-Huggins interaction parameters of different solvent-WPU pairs were yielded (Table II).

According to the rule "like dissolves like" (i.e., a solvent that has a similar polarity to the composites' matrix will usually be adsorbed by the substance very well), acetone vapor should be easily adsorbed by the composite CB/WPU-1 because the polarity of PEOG in WPU-1 is the highest as compared with the other two kinds of soft segments (Table I). The conclusion is supported by the Flory-Huggins interaction parameter of acetone-WPU-1, which is only 0.55 , much 

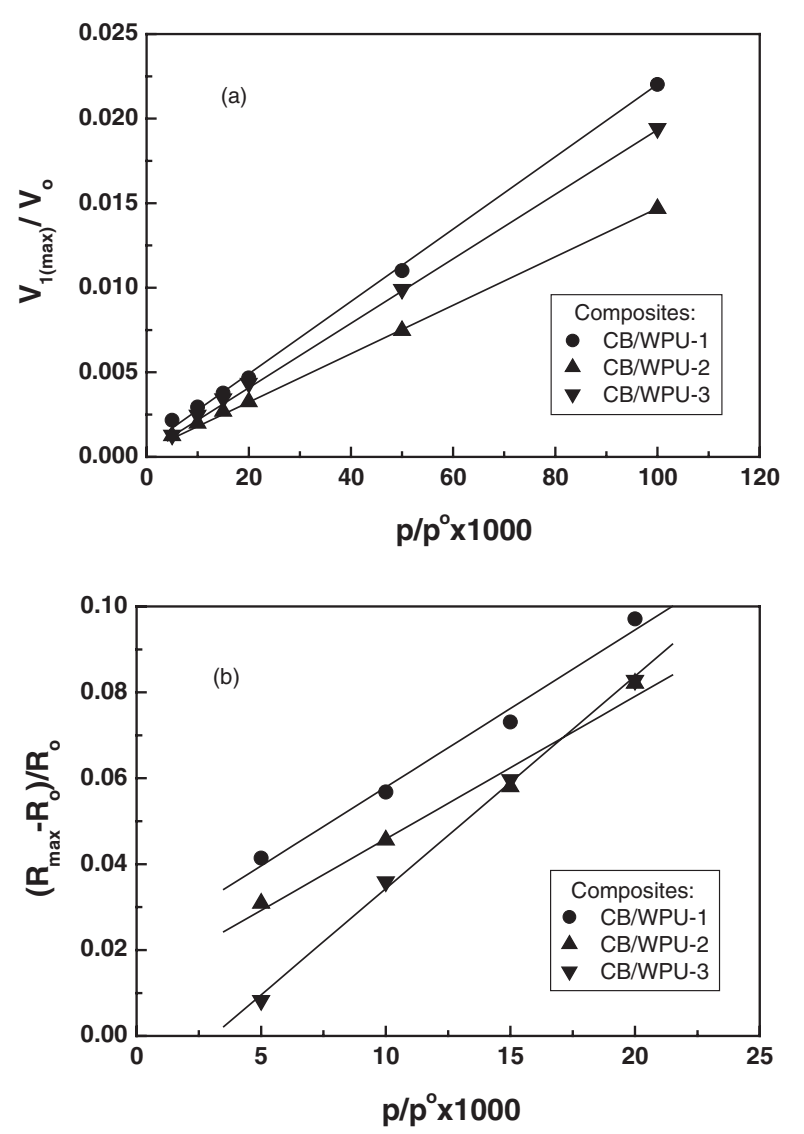

Figure 3. (a) Equilibrium adsorption capacity and (b) maximum responsivity of $\mathrm{CB} / \mathrm{WPU}$ composites in acetone vapor as a function of relative vapor pressure (temperature $=35^{\circ} \mathrm{C}$ ).

smaller than those of acetone-WPU-2 (0.96) and acetone-WPU-3 (0.67) (Table II). As a result, the equilibrium adsorption capacity of $\mathrm{CB} / \mathrm{WPU}-1$ composite in acetone vapor is the highest over the entire $p / p^{o}$ range of interests, while that of CB/WPU-2 the lowest (Figure 3(a)). In general, a higher adsorption quantity should result in higher sensitivity to the vapor, as predicted by the traditional swelling model. Evidently, it accounts for the highest maximum responsivity of CB/WPU-1 against acetone vapor (Figure 3(b)).

In the case of chloroform vapor, the Flory-Huggins interaction parameters of all the three solvent-WPU pairs are less than 0 (Table II). It means the interaction between chloroform molecules and each WPU is rather strong. Comparatively, the $\chi_{12}$ value of chloroform-WPU-1 is the highest, so that either the equilibrium adsorption capacity or the maximum responsivity of $\mathrm{CB} / \mathrm{WPU}-1$ in the vapor of chloroform is the lowest (Figure 4).

When the tests are conducted in non-polar benzene vapor, the Flory-Huggins interaction parameters of benzene-WPU-2 and benzene-WPU-3 are close to 0 , while that of benzene-WPU-1 is greater than 0 (Table II). It is reasonable as PEOG has the highest polarity and could not have strong interaction with the non-
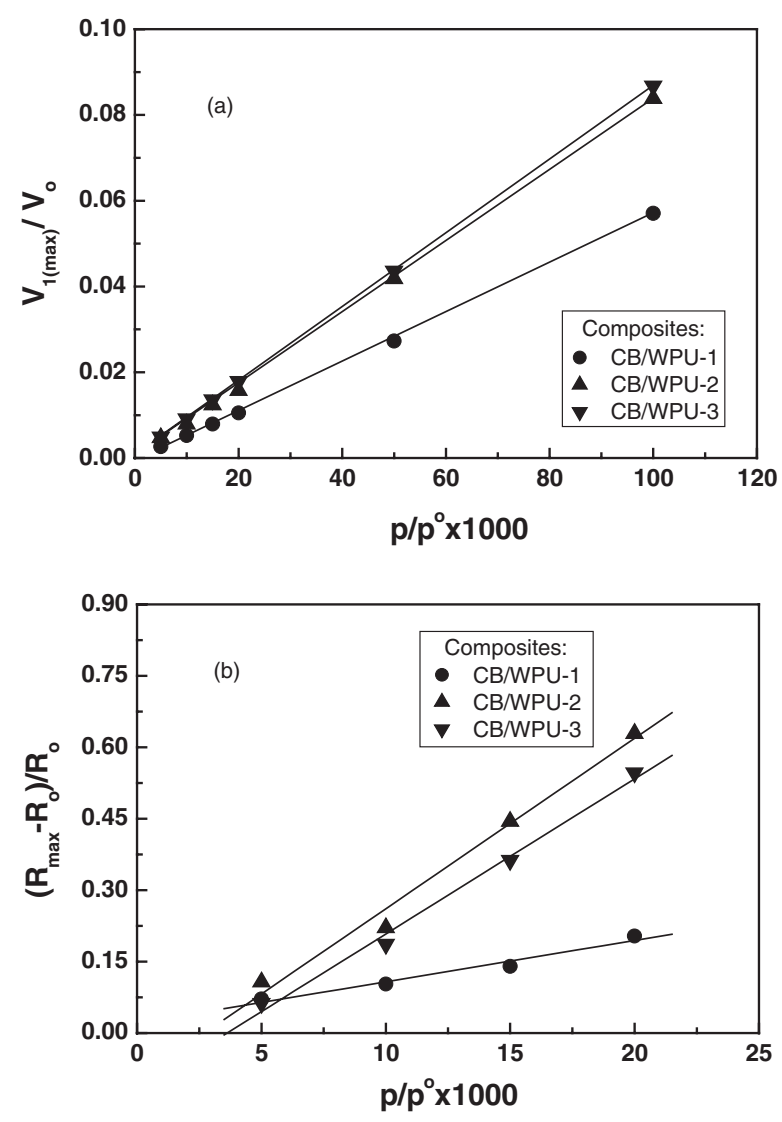

Figure 4. (a) Equilibrium adsorption capacity and (b) maximum responsivity of $\mathrm{CB} / \mathrm{WPU}$ composites in chloroform vapor as a function of relative vapor pressure (temperature $=35^{\circ} \mathrm{C}$ ).

polar solvent. Consequently, the equilibrium adsorption capacity and maximum responsivity of $\mathrm{CB} /$ WPU-1 in benzene vapor are lower than those of CB/WPU-2 and CB/WPU-3 composites (Figure 5).

It is noteworthy that in all the three solvent vapors CB/WPU-2 and CB/WPU-3 do not follow the swelling model as $\mathrm{CB} / \mathrm{WPU}-1$. Although the equilibrium adsorption capacity of $\mathrm{CB} / \mathrm{WPU}-3$ is higher than that of CB/WPU-2 (Figures 3a, 4a and 5a), the maximum responsivity of the former is mostly lower than that of the latter (Figures $3 b, 4 b$ and $5 b$ ). The result contradicts the law obeyed by CB/WPU-1. In principle, composites' gas sensitivity originates from disconnection of $\mathrm{CB}$ conductive networks driven by the movement of chain segments of surrounding swollen polymer. The above inconsistency implies that matrix swelling is not efficient enough to induce partial damage of the conduction paths. Therefore, interaction between swollen chain segments and CB particles has to be further investigated in the following.

\section{Selective Distribution}

Since the microstructure of polyurethane is characterized by microphase separation, a CB/WPU composite can be regarded as a ternary system consisting of 

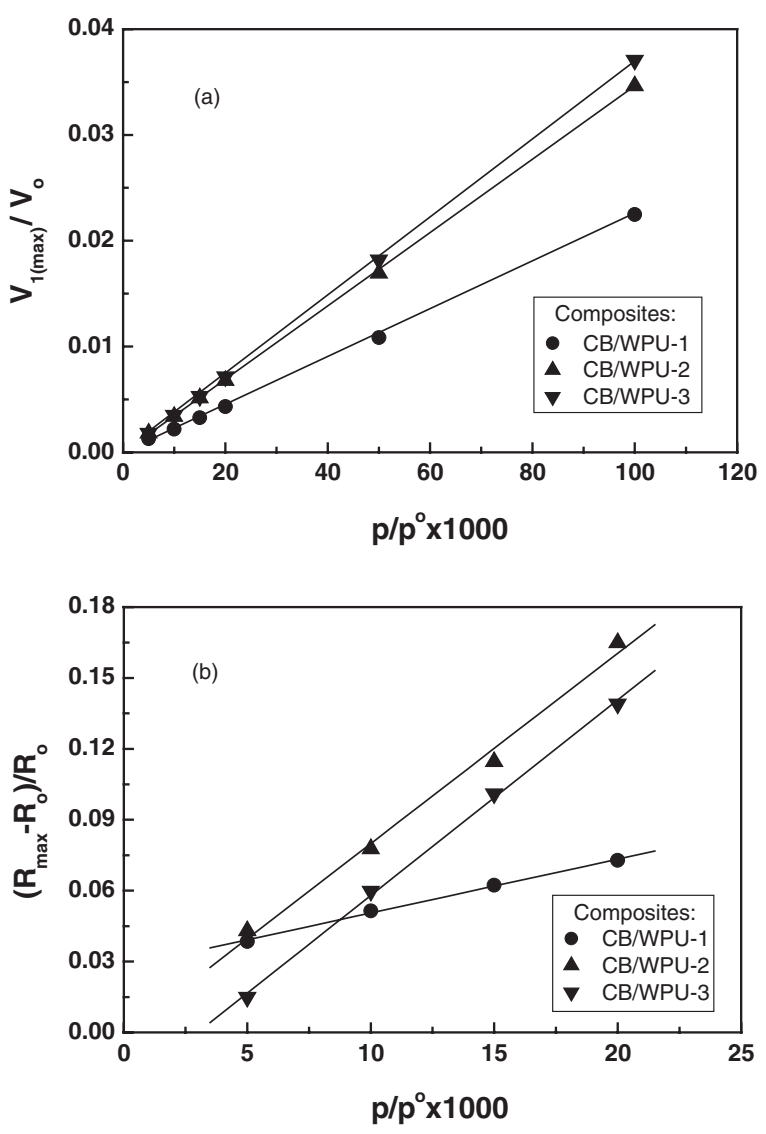

Figure 5. (a) Equilibrium adsorption capacity and (b) maximum responsivity of $\mathrm{CB} / \mathrm{WPU}$ composites in benzene vapor as a function of relative vapor pressure (temperature $=35^{\circ} \mathrm{C}$ ).

Table II. Flory-Huggins interaction parameters of the adsorbed solvent-WPU pairs

\begin{tabular}{crrr}
\hline Solvent & WPU-1 & WPU-2 & WPU-3 \\
\hline Acetone & 0.55 & 0.96 & 0.67 \\
Chloroform & -0.44 & -0.83 & -0.85 \\
Benzene & 0.50 & 0.06 & -0.01 \\
\hline
\end{tabular}

$\mathrm{CB}$, the microphase of the soft segments, and the microphase of the hard segments. The situation is similar to the conductive composites made by incorporation of CB into immiscible polymer blends. Sumita and co-workers proposed a wetting coefficient to deduce the preferred dispersion of a third component in binary blend: ${ }^{19}$

$$
\omega_{1-2}=\frac{\gamma_{3-2}-\gamma_{3-1}}{\gamma_{1-2}}
$$

where $\omega_{1-2}$ denotes the wetting coefficient of a blend consisting of components 1 and 2 , and $\gamma_{1-2}$ the interfacial free energy of the blend. $\gamma_{3-1}$ and $\gamma_{3-2}$ represent the interfacial free energies of the systems comprised by components 1 and 2 , and the third component (e.g., filler), respectively. $\omega_{1-2}>1$ suggests that the third component is located in the phase of component 1.
Table III. Surface free energies of WPU*, $\mathrm{CB}^{21}$ and the solvents

\begin{tabular}{|c|c|c|c|c|}
\hline \multicolumn{2}{|c|}{ Substances } & \multicolumn{3}{|c|}{$\gamma\left(\mathrm{mJ} / \mathrm{m}^{2}\right) \gamma^{p}\left(\mathrm{~mJ} / \mathrm{m}^{2}\right) \gamma^{d}\left(\mathrm{~mJ} / \mathrm{m}^{2}\right)$} \\
\hline \multirow{3}{*}{$\begin{array}{c}\text { Soft segments } \\
\text { of WPU }\end{array}$} & PEOG & 40.88 & 5.82 & 35.06 \\
\hline & PPG & 32.00 & 4.70 & 27.30 \\
\hline & PTMEG & 31.48 & 2.84 & 28.64 \\
\hline \multicolumn{2}{|c|}{$\begin{array}{l}\text { Hard segments } \\
\quad \text { of WPU }\end{array}$} & 26.32 & 5.20 & 21.12 \\
\hline \multirow[t]{2}{*}{ Filler } & $\mathrm{CB}$ & 42.20 & 0.30 & 41.90 \\
\hline & Chloroform & 27.50 & 1.60 & 25.90 \\
\hline \multirow[t]{2}{*}{ Solvents } & Benzene & 28.40 & 1.70 & 26.70 \\
\hline & Acetone & 23.30 & 6.80 & 16.50 \\
\hline
\end{tabular}

*The surface free energies of both the soft and hard segments of WPU were estimated as follows. Firstly, solubility parameter, $\delta\left(=\sqrt{\left.\left(\frac{\Sigma F_{d i}}{V}\right)^{2}+\frac{\Sigma F_{p i}^{2}}{V^{2}}+\frac{\Sigma E_{h i}}{V}\right)}\right.$, polar component of solubility parameter, $\delta_{p}\left(=\frac{\sqrt{\Sigma F_{p i}^{2}}}{V}\right)$, and surface free energy, $\gamma\left(=\left(\frac{P_{S}}{V}\right)^{4}\right)$, were calculated by using the group contribution method (where $F_{d i}$ denotes dispersive component of molar attraction constant of the repeat unit, $F_{p i}$ polar component of molar attraction constant of the repeat unit, $E_{h i}$ hydrogen bonding energy of the repeat unit, $P_{s}$ parachor of the repeat unit, and $V$ molar volume of the repeat unit). ${ }^{14}$ Then, polar component of surface free energy, $\gamma^{p}$, was obtained from the relationship: ${ }^{20} \frac{\gamma^{p}}{\gamma}=\left(\frac{\delta_{p}}{\delta}\right)^{2}$, while dispersive component of surface free energy, $\gamma^{d}$, was given by: $\gamma=\gamma^{d}+\gamma^{p}$.

In case of $-1<\omega_{1-2}<1$, the third component tends to be distributed at the interface of the 1-2 blend, while for $\omega_{1-2}<-1$, the third component is preferentially dispersed in the phase of component 2 . The interfacial free energy, $\gamma_{i-j}$, can be estimated from the harmonic mean method: ${ }^{20}$

$$
\gamma_{i-j}=\gamma_{i}+\gamma_{j}-4\left(\frac{\gamma_{i}^{d} \gamma_{j}^{d}}{\gamma_{i}^{d}+\gamma_{j}^{d}}+\frac{\gamma_{i}^{p} \gamma_{j}^{p}}{\gamma_{i}^{p}+\gamma_{j}^{p}}\right)
$$

where $\gamma_{i}^{d}$ or $\gamma_{j}^{d}$ stands for the dispersive component of surface free energy $\gamma_{i}$ or $\gamma_{j}$, and $\gamma_{i}^{p}$ or $\gamma_{j}^{p}$ denotes the polar component, respectively.

By using the wetting coefficient, therefore, we might know that the majority of $\mathrm{CB}$ particles in the WPU matrix are distributed in either the microphase of the soft segments or the microphase of the hard segments. Accordingly, the wetting coefficient $\omega_{W P U(s)-W P U(h)}$ concerning CB is calculated:

$$
\omega_{W P U(s)-W P U(h)}=\frac{\gamma_{C B-W P U(h)}-\gamma_{C B-W P U(s)}}{\gamma_{W P U(s)-W P U(h)}}
$$

where the subscripts $W P U(s)$ and $W P U(h)$ denote the soft and hard segments of WPU, respectively.

Table III lists useful data for the calculation, based on which $\omega_{W P U(s)-W P U(h)}$ was estimated (Table IV). From the aforesaid criteria, it is obvious that the CB 
Table IV. Wetting coefficients concerning preferred distribution of CB in WPU microphases

\begin{tabular}{lccc}
\hline Composites & CB/WPU-1 & CB/WPU-2 & CB/WPU-3 \\
\hline$\omega_{W P U(s)-W P U(h)}$ & 1.61 & 5.23 & 3.65 \\
\hline
\end{tabular}

Table V. Wetting coefficients concerning preferred adsorption of the solvents in WPU microphases

\begin{tabular}{cccc}
\hline WPU & Chloroform & Benzene & Acetone \\
\hline WPU-1 & -0.40 & -0.28 & -1.71 \\
WPU-2 & 1.01 & 1.24 & -2.78 \\
WPU-3 & 1.04 & 1.13 & -2.25 \\
\hline
\end{tabular}

particles tend to stay within the microphase of the soft segments of WPU in all the three kinds of CB/WPU composites.

To analyze the efficiency of swelling induced electrical resistance variation, the preferential site of WPU microphases for solvent uptake should also be known. Similarly, the corresponding wetting coefficients are acquired from:

$$
\omega_{W P U(s)-W P U(h)}^{\prime}=\frac{\gamma_{\text {solvent-WPU(h) }}-\gamma_{\text {solvent-WPU(s) }}}{\gamma_{W P U(s)-W P U(h)}}
$$

Table V collects the values of $\omega_{W P U(s)-W P U(h)}^{\prime}$ of the three kinds of WPU in different solvent vapors. In the case of chloroform and benzene, the wetting coefficients considered for WPU-2 and WPU-3 are greater than 1 , suggesting that the solvents preferentially attack the soft segments of the matrices. As CB particles are selectively located in the microphase of the soft segments, the swelling resulting from adsorption of both chloroform and benzene should be very efficient to break down the conduction $\mathrm{CB}$ networks. This is true especially for CB/WPU-2, because it has the highest $\omega_{W P U(s)-W P U(h)}($ Table IV). As a result, the maximum responsivity of $\mathrm{CB} / \mathrm{WPU}-2$ against the vapors of chloroform and benzene is superior to that of CB/WPU-3. With respect to WPU-1, the two solvents tend to immigrate into the interface of the microphases of soft and hard segments $\left(-1<\omega_{W P U(s)-W P U(h)}^{\prime}<\right.$ 1 , see Table V). It means the soft segments of WPU-1 cannot be efficiently swollen like the other two WPUs, which accounts for the lowest maximum responsivity of CB/WPU-1 in chloroform and benzene vapors.

For the tests carried out in acetone vapor, the values of $\omega_{W P U(s)-W P U(h)}^{\prime}$ of all the three WPUs are less than -1 . It manifests that the solvent prefers to attack their polar hard segments, which coincides with the analysis based on polarity. In polyurethane, hard segments play the role of physical crosslinking. When their mobility is increased upon exposure to a polar solvent, their restriction on the soft segments would be re-

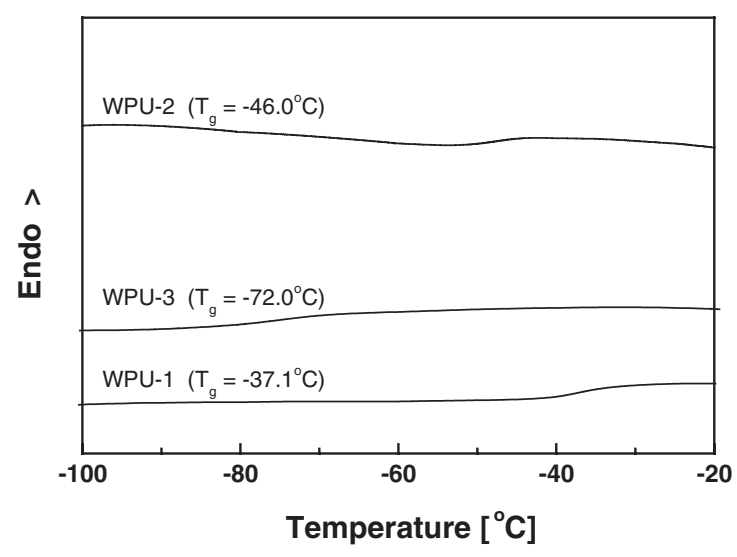

Figure 6. DSC heating traces of the WPUs.

leased and hence the CB fillers adjacent to the soft segments can be disconnected exhibiting increased resistance of the composite. Since $\omega_{W P U(s)-W P U(h)}$ of $\mathrm{CB} / \mathrm{WPU}-1$ is slightly greater than 1 and much smaller than the values of CB/WPU-2 and CB/WPU-3 (Table IV), it can be deduced that the fillers in $\mathrm{CB} /$ WPU-1 might be more easily influenced by the movement of the soft segments driven by the swollen hard segments. That is, CB/WPU-1 shows the highest maximum responsivity to acetone vapor than the rest two kinds of composites.

\section{Rate of Response}

Although vapor adsorption and sensitivity of the WPU composites have been analyzed in the above two sections, the effect of the soft segments of WPU on rate of response of the composites still remains unknown. Some earlier works indicated that the rate of solvent vapor diffusion into polymer matrices of composites predominates the response kinetics. ${ }^{4,22,23} \mathrm{Gen}$ erally, a Fickian diffusion of solvents into polymers (case I) (perceived in the rubbery state) is relaxation dependent. A non-Fickian diffusion (case II) is characterized by a sharp concentration profile in glassy polymers. Figure 6 shows the DSC analysis of glass transition of the three WPUs. It is seen that their glass transition temperatures are far lower than room temperature. As the response tests were conducted at $35^{\circ} \mathrm{C}$, Fickian model should be applied in the subsequent discussion. On the other hand, the DSC study also demonstrates that no crystallization takes place in the WPUs. Hence the effect of crystallization on diffusion can be excluded from consideration.

The normalized adsorbance of the composites in low concentration chloroform vapor is plotted in Figure $7 \mathrm{a}$ as a function of square root of time. From the slopes of the linear portions of the plots at the initial stage, $k$, the diffusion coefficient, $D$, is yielded:

$$
D=\frac{\pi}{16}(k L)^{2}
$$



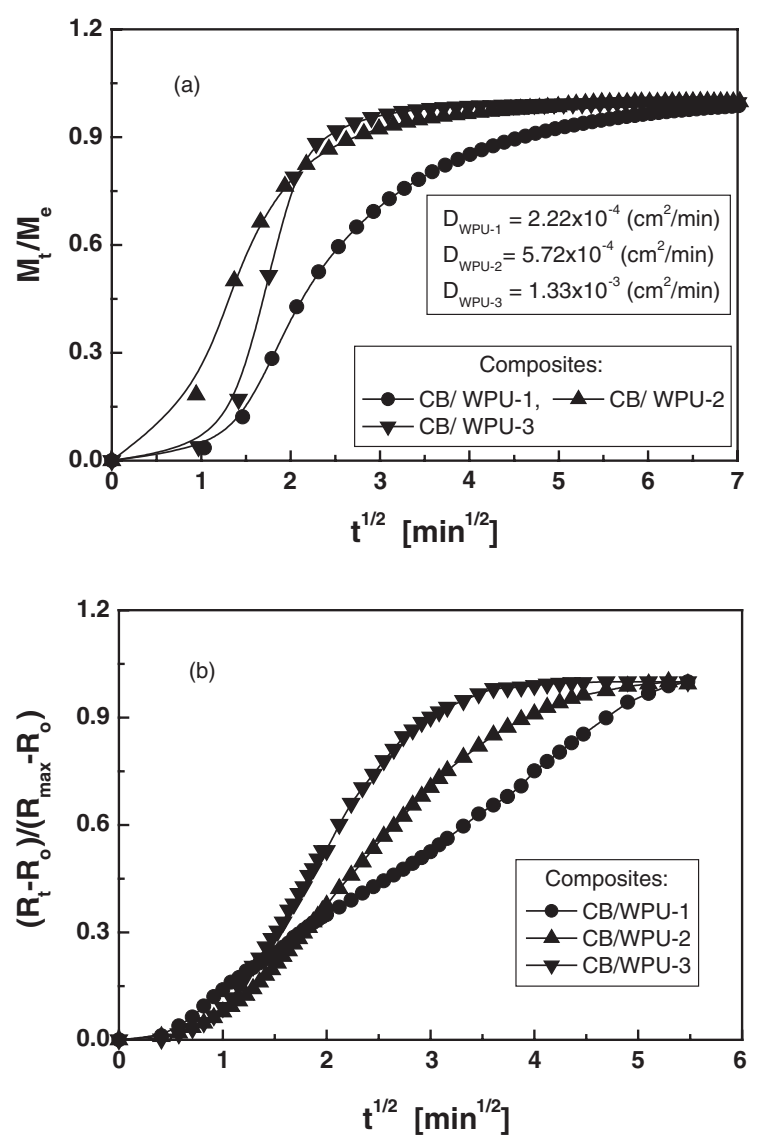

Figure 7. (a) Normalized adsorbance and (b) relative variation in resistance of $\mathrm{CB} / \mathrm{WPU}$ composites in chloroform vapor as a function of square root of time, $\sqrt{t}$ (temperature $=35^{\circ} \mathrm{C}$; $p / p^{o}=0.01$; specimen thickness of $\mathrm{CB} / \mathrm{WPU}-1, \mathrm{CB} / \mathrm{WPU}-2$ and $\mathrm{CB} / \mathrm{WPU}-3=55.4,53.9$ and $55.5 \mu \mathrm{m}$, respectively).

where $L$ denotes the sample film thickness. By examining the calculated diffusion coefficients in Figure $7 \mathrm{a}$, it is clear that the composites give the following rank: $\mathrm{CB} / \mathrm{WPU}-3>\mathrm{CB} / \mathrm{WPU}-2>\mathrm{CB} / \mathrm{WPU}-1$, which is in reversed sequence of the glass transition temperatures of the WPUs. The results prove that the flexibility of the WPU soft segments is a controlling factor, which agrees with the molecular mechanism involved in Fickian diffusion.

Figure $7 \mathrm{~b}$ presents the time related response behavior of the CB/WPU composites in chloroform vapor drawn in the way similar to Figure $7 \mathrm{a}$, so that the slopes of the linear portions of the curves can be used as a measure of the rate of response. Obviously, CB/ WPU-3 has the highest rate of response, while $\mathrm{CB}$ / WPU-1 the lowest. Besides the rate of solvent diffusion, it is believed that the selective adsorption of the solvent should also take the responsibility. As implied by the data in Table V, chloroform preferentially interacts with the soft segments/hard segments microphases interface of WPU-1 as the wetting coefficient is -0.4 . In addition, the solvent tends to immigrate directly into the microphases of soft segments of

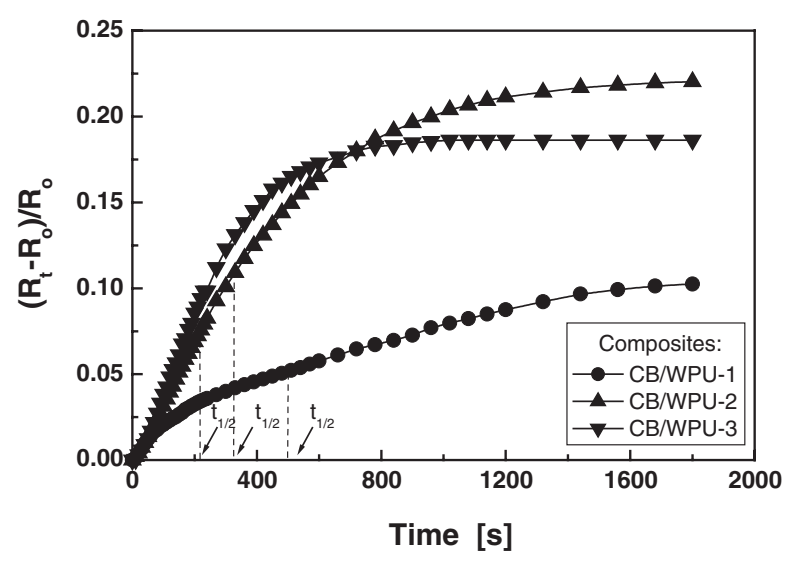

Figure 8. Typical electrical responses of CB/WPU composites against chloroform vapor at a relative pressure of 0.01 (temperature $\left.=35^{\circ} \mathrm{C}\right) . t_{1 / 2}$ represents the response time.

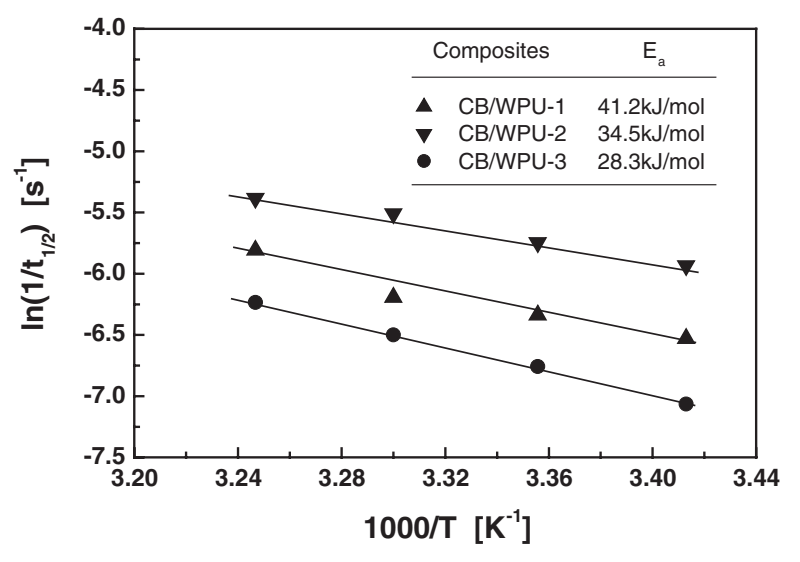

Figure 9. Arrhenius plots of $\mathrm{CB} / \mathrm{WPU}$ composites in response to chloroform vapor $\left(p / p^{o}=0.01\right)$.

WPU-2 and WPU-3. Due to the fact that CB is preferentially distributed inside the microphase of the WPU soft segments, the resistance variation of $\mathrm{CB} /$ WPU-1 has to proceed more slowly than those of $\mathrm{CB} / \mathrm{WPU}-2$ and $\mathrm{CB} / \mathrm{WPU}-3$.

On the other hand, our previous works found that the composites' gas sensitivity is controlled by structural relaxation of the materials. ${ }^{24,25}$ The response time can be correlated with absolute temperature by Arrhenius equation, and the estimated activation energy reflects mobility of the fillers involved in the solvent induced expansion of the surrounding polymer. Hereinafter the response kinetics of CB/WPU composites is also treated by the same approach. That is, electrical responses of the composites against chloroform vapor were measured at different temperatures $\left(20-35^{\circ} \mathrm{C}\right)$ and then the response times (define as the times to reach half of the maximum responsivity, see Figure 8) were determined, giving a series of Arrhenius plots and the corresponding activation energies, $E_{a}$ (Figure 9). The lowest $E_{a}$ value of CB/WPU3 can be explained by the greatest mobility of CB par- 
ticles provided by the swollen WPU-3, which contains soft segments with the highest flexibility. The CB/ WPU-1 composite exhibits the highest $E_{a}$ because much more energy has to be consumed to provide $\mathrm{CB}$ particles with the same mobility as in CB/WPU-3. These again confirm the previous consideration of the role of the WPU soft segments.

\section{CONCLUSIONS}

By evaluating gas sensitivity of three kinds of CB/ WPU composites, in which the soft segments of each matrix polymer are different in flexibility and polarity, the main influencing factors have been studied in detail. Owing to the specific structures of the matrices, the maximum responsivity of the composites is dependent not only on solvent-polymer interaction but also on selective adsorption of solvent. The traditional swelling model failed to explain some experimental phenomena because of the reduced efficiency of swelling in inducing disconnection of the conduction paths throughout the composites. The rate of electrical response is closely related to the flexibility of the soft segments of WPU and preferential localization of solvent in the matrix polymer as well. The composite with its matrix containing more flexible soft segments that attract direct attack of solvent molecules exhibits faster response manner.

Acknowledgment. The authors would like to thank the financial supports of the Natural Science Foundation of China (Grant 50133020), the Team Project of the Natural Science Foundation of Guangdong (Grant 20003038) and the Talent Training Program Foundation of the Higher Education Department of Guangdong Province.

\section{REFERENCES}

1. B. Lundberg and B. Sundqvist, J. Appl. Phys., 60, 1074 (1986).

2. P. Talik, M. Zabkowska-Waclawek, and W. Waclawek, J. Mater. Sci., 27, 6807 (1992).
3. A. Marquez, J. Uribe, and R. Cruz, J. Appl. Polym. Sci., 66, 2221 (1997).

4. J. F. Feller and Y. Grohens, Sens. Actuators, B, 97, 231 (2004).

5. K. J. Albert, N. S. Lewis, C. L. Schauer, G. A. Sotzing, S. F. Stitzel, and T. P. Vaid, Chem. Rev., 100, 2595 (2000).

6. M. Narkis, S. Srivastava, R. Tchoudakov, and O. Bereuer, Synth. Met., 113, 29 (2000).

7. F. Zee and J. W. Judy, Sens. Actuators, B, 72, 120 (2001).

8. J. W. Hu, M. W. Li, M. Q. Zhang, D. S. Xiao, G. S. Cheng, and M. Z. Rong, Macromol. Rapid. Commun., 24, 889 (2003).

9. S. G. Chen, J. W. Hu, M. Q. Zhang, M. W. Li, and M. Z. Rong, Carbon, 42, 645 (2004).

10. S. G. Chen, J. W. Hu, M. Q. Zhang, and M. Z. Rong, Sens. Actuators, B, 105, 187 (2005).

11. J. W. Hu, S. G. Chen, M. Q. Zhang, M. W. Li, and M. Z. Rong, Mater. Lett., 58, 3606 (2004).

12. S. G. Chen, J. W. Hu, M. Q. Zhang, M. Z. Rong, and Q. Zheng, Sens. Actuators, B, 113, 361 (2006).

13. S. G. Chen, X. L. Hu, J. Hu, M. Q. Zhang, M. Z. Rong, and Q. Zheng, Sens. Actuators, B, (in press).

14. D. W. van Krevelen, "Properties of Polymers," Elsevier Science, Amsterdam, 1976.

15. J. H. Chen and N. Tsubokawa, Polym. J., 32, 729 (2000).

16. X. M. Dong, R. W. Fu, M. Q. Zhang, B. Zhang, J. R. Li, and M. Z. Rong, Carbon, 42, 2551 (2004).

17. P. J. Flory, "Principles of Polymer Chemistry," Cornell University Press, New York, 1953.

18. O. Rodrígueza, F. Fornasieroa, A. Arcea, C. J. Radke, and J. M. Prausnitz, Polymer, 44, 6323 (2003).

19. M. Sumita, K. Sakata, S. Asai, K. Miyasaka, and H. Nakagawa, Polym. Bull., 25, 265 (1991).

20. S. Wu, "Polymer Interface and Adhesion," Marcel Dekker Inc., New York, 1982.

21. M. Q. Zhang, G. Yu, H. M. Zeng, H. B. Zhang, and Y. H. Hou, Macromolecules, 31, 6724 (1998).

22. J. F. Feller, D. Langevin, and S. Marai, Synth. Met., 144, 81 (2004).

23. A. Carrillo, I. R. Martín-Domínguez, D. Glossman, and A. Márquez, Sens. Actuators, A, 119, 157 (2005).

24. S. G. Chen, J. W. Hu, M. Q. Zhang, M. Z. Rong, and Q. Zheng, J. Mater. Sci., 40, 2065 (2005).

25. J. R. Li, J. Wang, J. R. Xu, M. Q. Zhang, M. Z. Rong, and Q. Zheng, Compos. Sci. Technol., (in press). 